\title{
polR, a pathway-specific transcriptional regulatory gene, positively controls polyoxin biosynthesis in Streptomyces cacaoi subsp. asoensis
}

\begin{abstract}
Correspondence
Huarong Tan

tanhr@sun.im.ac.cn

Zixin Deng

zxdeng@sjtu.edu.cn
\end{abstract}

Received 24 February 2009

Accepted 6 March 2009

\author{
Rui Li, ${ }^{1} \dagger$ Zhoujie Xie, ${ }^{1} \dagger$ Yuqing Tian, ${ }^{1}$ Haihua Yang, ${ }^{1}$ Wenqing Chen, ${ }^{2}$ \\ Delin You, ${ }^{2}$ Gang Liu, ${ }^{1}$ Zixin Deng ${ }^{2}$ and Huarong Tan ${ }^{1}$
${ }^{1}$ State Key Laboratory of Microbial Resources, Institute of Microbiology, Chinese Academy of Sciences, Beijing 100101, China
${ }^{2}$ Laboratory of Microbial Metabolism and School of Life Science and Biotechnology, Shanghai Jiaotong University, Shanghai 200030, China

\begin{abstract}
The polyoxin (POL) biosynthetic gene cluster $(\mathrm{pol})$ was recently cloned from Streptomyces cacaoi subsp. asoensis. A 3.3 kb DNA fragment carrying an obvious open reading frame (polR), whose deduced product shows sequence similarity to SanG of Streptomyces ansochromogenes and PimR of Streptomyces natalensis, was revealed within the pol gene cluster. Disruption of polR abolished POL production, which could be complemented by the integration of a single copy of polR into the chromosome of the non-producing mutant. The introduction of an extra copy of polR in the wild-type strain resulted in increased production of POLs. The transcription start point (tsp) of polR was determined by S1 mapping. Reverse transcriptase PCR experiments showed that PolR is required for the transcription of 18 structural genes in the pol gene cluster. Furthermore, we showed that $p o / C$ and $p o / B$, the respective first genes of two putative operons ( $p o / C-p o / Q 2$ and $p o / A-p o / B)$ consisting of 16 and 2 of these 18 genes, have similar promoter structures. Gel retardation assays indicated that PoIR has specific DNA-binding activity for the promoter regions of po/C and polB. Our data suggest that PolR acts in a positive manner to regulate POL production by activating the transcription of at least two putative operons in the pol gene cluster.
\end{abstract}

\section{INTRODUCTION}

Streptomycetes are Gram-positive filamentous soil bacteria with a complex life cycle, and are also well known for their capacity to produce a vast majority of secondary metabolites, many of which are commercially important antibiotics widely used in medicine and agriculture. Studies on the model organism Streptomyces coelicolor and other antibiotic-producing streptomycetes have revealed that regulation of antibiotic biosynthesis is a complex process (Hopwood et al., 1995; Paradkar et al., 1998). Antibiotic production in Streptomyces species is generally dependent on the growth phase, and involves the expression of clustered biosynthetic genes. Pathway-specific regulatory genes are usually linked to these gene clusters, and many pathway-specific regulators belong to a protein family called SARPs (Streptomyces antibiotic regulation proteins) (Wietzorrek \& Bibb, 1997). Members of this family share

†These authors contributed equally to this work.

Abbreviations: NIK, nikkomycin; POL, polyoxin; SARP, Streptomyces antibiotic regulation protein; tsp, transcription start point.

The GenBank/EMBL/DDBJ accession number for the sequence reported in this paper is EU158805. an N-terminal OmpR-type DNA-binding domain, usually followed by a bacterial transcriptional activation domain (BTAD) (Tanaka et al., 2007). Examples of this family include the positive regulators ActII-ORF4 and RedD from $S$. coelicolor, which regulate the biosynthesis of actinorhodin and undecylprodigiosin, respectively (Arias et al., 1999; Narva \& Feitelson, 1990), DnrI from Streptomyces peucetius, which controls the production of daunorubicin (Stutzman-Engwall et al., 1992), and CcaR from Streptomyces clavuligerus, which regulates the production of cephamycin and clavulanic acid (Perez-Llarena et al., 1997). Significantly, recent characterization of several antibiotic pathways has led to the identification of a set of novel transcriptional regulators. This unique group of transcriptional regulators has extra domains in addition to the conserved core of SARP-family proteins, and has a relatively large size compared with those of the typical SARP family. PimR and SanG, positive regulators of pimaricin and nikkomycin (NIK) production, respectively, belong to this group (Anton et al., 2004; Liu et al., 2005).

Polyoxins (POLs), a group of nucleoside peptide antifungal antibiotics, were isolated from the culture broths of Streptomyces cacaoi subsp. asoensis. These antibiotics show 
high activity against phytopathogenic fungi. At least 12 components of POLs (designated alphabetically Pol-A to -L) have been reported (Isono et al., 1969). Since the POLs have a similar structure to UDP- $N$-acetylglucosamine, they are competitive inhibitors of chitin synthase and have proved to be excellent fungicides (Endo et al., 1970). The biosynthesis of POLs has been extensively investigated in vivo and a possible biosynthetic pathway was proposed by Isono and coworkers using radiolabelled precursor feeding experiments (Isono, 1988). POLs have a similar nucleoside moiety to that of NIKs (Müller et al., 1981). NIK biosynthesis gene clusters (nik) have been cloned from Streptomyces tendae and Streptomyces ansochromogenes (Bormann et al., 1996; Chen et al., 2000). The biosynthetic pathway and the regulation mechanism of NIK biosynthesis have been widely studied (Bruntner et al., 1999; Lauer et al., 2000, 2001; Liu et al., 2005). Considering the structural similarity between POL and NIK (Fig. 1a), it is likely that some similar gene homologues could be shared for their biosynthesis. Based on this idea, the pol gene cluster from S. cacaoi subsp. asoensis was cloned recently (Chen et al., 2009). The organization of genes with potential functions in the regulation or synthesis of polyoxins is shown in Fig. 1(b). Boundaries of the pol gene cluster were determined by gene disruption and polyoxin biosynthesis in a heterologous host. Twenty genes (polR, polY, polC-Q2, polA and polB) were defined as necessary for polyoxin production. The deduced functions for most of these genes correlated well with the biosynthetic pathway of polyoxins proposed by Isono and coworkers (Isono, 1988). Remarkably, the pol cluster contains two potential regulatory genes, polR and polY, that are SARP-encoding genes. polR is the homologue of $\operatorname{san} G$, which is a regulatory gene for NIK biosynthesis. In this paper, we describe detailed characterization of polR and demonstrate its role as a positive regulator of polyoxin biosynthesis in S. cacaoi subsp. asoensis.

\section{METHODS}

Strains, media and chemicals. S. cacaoi subsp. asoensis AS4.1602, a natural POL producer, was obtained from CGMCC (China General Microbiological Culture Collection Centre). Alternaria longipes was used as indicator strain for POL bioassay. Escherichia coli DH5 $\alpha$ was used as a host for propagating plasmids. E. coli ET12567(pUZ8002) was used for transferring DNA from E. coli to Streptomyces by conjugation (Liu et al., 2005). E. coli BL21(DE3) (Novagen) was used as a host for the overexpression of PolR.

S. cacaoi subsp. asoensis was grown on mannitol/soya (MS) medium at $28{ }^{\circ} \mathrm{C}$ (Kieser et al., 2000). For genomic DNA extraction, S. cacaoi subsp. asoensis was grown in YEME medium, which was also used as a seed medium (Kieser et al., 2000). SP medium (3\% mannitol, $1 \%$ soluble starch, $0.75 \%$ yeast extract and $0.5 \%$ soy peptone, $\mathrm{pH} 6.0$ ) was used for POL production (Bruntner et al., 1999).

When necessary, antibiotics were used at the following concentrations: apramycin, $10 \mu \mathrm{g} \mathrm{ml}^{-1}$ in YEME or MS for S. cacaoi subsp.

\section{(a)}

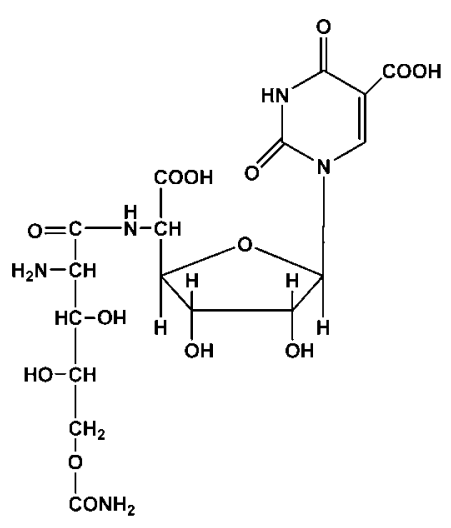

Polyoxin D

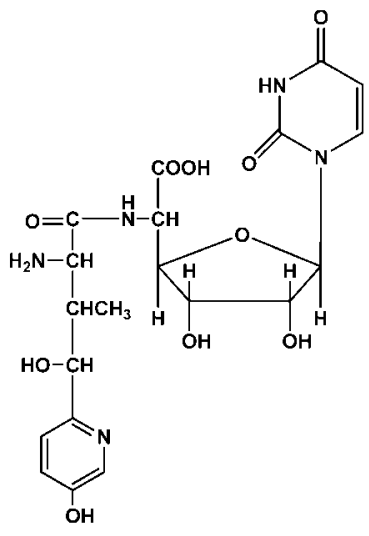

Nikkomycin Z

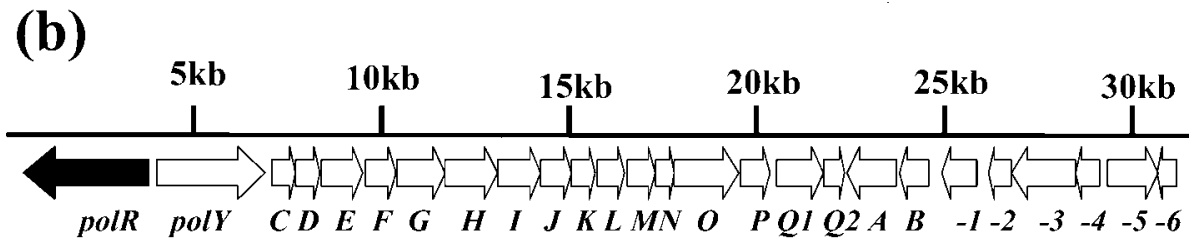

Fig. 1. (a) Chemical structures of polyoxin $D$ and the structurally related nikkomycin $Z$. These two antibiotics share a similar nucleoside moiety. (b) Organization of the POL biosynthesis gene cluster. Each arrow indicates a separate ORF and orientation of transcription. The black arrow indicates polR, a putative pathway-specific regulatory gene described in this paper. 
asoensis, $100 \mu \mathrm{g} \mathrm{ml}^{-1}$ in LB for E. coli; kanamycin, $10 \mu \mathrm{g} \mathrm{ml}^{-1}$ in YEME or MS for S. cacaoi subsp. asoensis, $100 \mu \mathrm{g} \mathrm{ml}^{-1}$ in LB for $E$. coli.

Plasmids and DNA manipulation. pBluescript $\mathrm{KS}(+)$ was used for routine DNA cloning. pUC119: : neo was the source of the kanamycin resistance gene. The E. coli-Streptomyces shuttle vector pKC1139, which contains a Streptomyces temperature-sensitive origin of replication from pSG5 (Bierman et al., 1992), was used to construct a recombinant plasmid for gene disruption. pSET152, which can integrate into the Streptomyces chromosome by site-specific recombination at the phage $\phi \mathrm{C} 31$ attachment site (attB) (Bierman et al., 1992; Kuhstoss et al., 1991), was used to create a recombinant plasmid for introducing a single copy of polR into Streptomyces. pET28a (Novagen) was used to construct the polR expression plasmid.

Plasmids and chromosomal DNA were isolated by standard techniques (Kieser et al., 2000). Conjugal transfer from E. coli ET12567(pUZ8002) to S. cacaoi subsp. asoensis was carried out as described by Kieser et al. (2000). The digoxigenin-11-dUTP labelling and detection kit (Roche Diagnostics) was used in Southern blot experiments according to the protocols provided by the manufacturer.

DNA sequence analysis. DNA sequences were analysed with the NCBI worldwide web BLAST and Frameplot 2.3 server. Protein domain architectures were analysed by using the SMART, Pfam and CDD databases and software tools.

Construction of polR disruption mutants. Disruption of $p o l R$ was performed by gene replacement via homologous recombination. For this purpose, a $1885 \mathrm{bp}$ polR fragment was amplified from $S$. cacaoi subsp. asoensis genomic DNA by PCR using primers DR Forward and DR Reverse (Table 1). PCR amplification was carried out using KOD plus DNA polymerase (TOYOBO Co.). The resulting DNA fragment was ligated into the EcoRV site of pKC1139 to generate pKC1139::polR. The kanamycin-resistance gene (neo) was obtained from pUC119:: neo after digestion with both BamHI and KpnI, blunted by mung bean nuclease and ligated into the SmaI site in pKC1139::polR to give pKC1139::polR:: neo, in which the neo gene has the same orientation as polR. The recombinant plasmid, pKC1139:: polR:: neo, was designated pDR101.

pDR101 was first passed through E. coli ET12567(pUZ8002) and then introduced into S. cacaoi subsp. asoensis via conjugation (Kieser et al., 2000). The resulting exconjugants were incubated on MS agar to form spores. Grey spores were harvested and spread on MS agar containing kanamycin. After incubation at $40{ }^{\circ} \mathrm{C}$ for 3 days, colonies that conferred kanamycin resistance $\left(\operatorname{Kan}^{\mathrm{R}}\right)$ and apramycin sensitivity $\left(A p r{ }^{S}\right)$ were selected and further confirmed as polR disruption mutants of polR by Southern blotting analysis. The polR disruption mutant selected for further study was named strain DMR.

Complementation of the polR mutant. For complementation analysis, a $3.8 \mathrm{~kb}$ DNA fragment containing intact polR and its flanking sequence was amplified by PCR using polR Forward and polR Reverse primers (Table 1). The amplified fragment was inserted into the EcoRV site of pSET152 to generate pSET152::polR, which was designated pSER.

Subsequently, pSER was integrated into the chromosomal $\phi \mathrm{C} 31$ attB site of the polR disruption mutant by conjugation (Kieser et al., 2000). pSER was also used for the overexpression of polR in the wild-type strain.

Bioassay and HPLC analysis of POLs. Spores of S. cacaoi subsp. asoensis were inoculated in YEME. The cultures were grown at $28{ }^{\circ} \mathrm{C}$ on a rotary shaker (220 r.p.m.) for $36 \mathrm{~h}$ and used as seed cultures.
Mycelia from the seed culture were inoculated into SP medium for POL production.

POL production was measured by a disk agar diffusion method using A. longipes as indicator strain. A $100 \mu \mathrm{l}$ sample of culture filtrate of each strain was used in the bioassay. POL production was further confirmed by HPLC analysis. Because POLs consist of at least 11 active components with similar structure (Isono, 1988), it is difficult to separate and identify all the components by HPLC at the same time. Of all the POL components reported, only POL-D is commercially available (Calbiochem cat. no. 529313), so we set up a separation system for detecting POL-D. An Agilent 1100 HPLC system equipped with an SB-C18 reverse-phase column was used. A mixure of $95 \%$ solvent A ( $10 \mathrm{mM}$ tetrabutylammonium hydroxide, adjusted to $\mathrm{pH} 4.0$ using glacial acetic acid) and $5 \%$ solvent $\mathrm{B}$ (acetonitrile) was applied with a constant flow rate of $1 \mathrm{ml} \mathrm{min}^{-1}$ for $20 \mathrm{~min}$. The detection wavelength was $290 \mathrm{~nm}$. Under these conditions POL-D had a retention time of $10 \mathrm{~min}$.

S1 nuclease mapping. RNAs for S1 nuclease mapping were isolated from S. cacaoi subsp. asoensis after incubation in SP liquid medium for $48 \mathrm{~h}$. Mycelia were collected, quickly frozen in liquid nitrogen and ground into a fine white powder. RNA samples were then extracted using Trizol reagent (Invitrogen) according to the manufacturer's protocol. S1 nuclease mapping was performed as described previously (Liu et al., 2005).

All the primers used for S1 nuclease mapping are listed in Table 1. For polR, the hybridization probe was prepared by PCR using the unlabelled oligonucleotide primer S-polR-AN and labelled primer SpolR-SE. Primer S-polR-SE was labelled at the $5^{\prime}$ end with $\left[\gamma_{-}{ }^{32} \mathrm{P}\right] \mathrm{ATP}$ using T4 polynucleotide kinase. For polC, the probe was generated by PCR with the unlabelled primer S-polC-AN and labelled primer SpolC-SE. For polB, the probe was amplified using the unlabelled primer S-polB-AN and labelled primer S-polB-SE. The DNA sequencing ladders were generated using the fmol DNA cycle sequencing kit (Promega) with the corresponding labelled primers. Protected DNA fragments were analysed by electrophoresis on $6 \%$ polyacrylamide gels containing $7 \mathrm{M}$ urea.

Reverse transcriptase PCR (RT-PCR). RNA samples were isolated from wild-type and DMR strains grown in SP liquid medium at $28{ }^{\circ} \mathrm{C}$ for $48 \mathrm{~h}$, using Trizol reagent (Invitrogen). The transcription profiles of pol genes were compared between the wild-type and polR mutant strain by a semiquantitative RT-PCR analysis.

The Qiagen One-Step RT-PCR kit was used with primers specific for each gene and designed to produce PCR products ranging from 300 to $600 \mathrm{bp}$. Forward and reverse primers are shown in Table 1. Each primer pair was first tested using chromosomal DNA as a template. Absence of DNA contamination in the RNA samples was assessed by PCR. Reaction mixtures contained 6 pmol of each primer and $150 \mathrm{ng}$ RNA in a total volume of $20 \mu \mathrm{l}$. RT-PCR conditions were as follows: first-strand cDNA synthesis, $50{ }^{\circ} \mathrm{C}$ for $30 \mathrm{~min}$ and $55{ }^{\circ} \mathrm{C}$ for $30 \mathrm{~min}$ followed by $95{ }^{\circ} \mathrm{C}$ for $15 \mathrm{~min}$; amplification, 27 or 32 cycles of $94{ }^{\circ} \mathrm{C}$ for $45 \mathrm{~s}, 50{ }^{\circ} \mathrm{C}$ to $60{ }^{\circ} \mathrm{C}$ (depending on the set of primers used) for $45 \mathrm{~s}$, and $72{ }^{\circ} \mathrm{C}$ for $1 \mathrm{~min}$; final extension, $72{ }^{\circ} \mathrm{C}$ for $10 \mathrm{~min}$. Twentyseven cycles of PCR were routinely employed; whenever no product was detected, 32 cycles of PCR were repeated to detect transcripts present at low levels. Products were detected by $1 \%$ agarose gel electrophoresis and visualized by staining with ethidium bromide.

Expression and purification of recombinant PolR. The coding region of polR was amplified by PCR from $S$. cacaoi subsp. asoensis genomic DNA using the primers PolRN and PolRC, containing NdeI and EcoRI sites respectively. The PCR product was digested with NdeI and EcoRI and cloned into the corresponding sites of pET28a to generate expression plasmid pET28a::polR. The recombinant plas- 
Table 1. Primers used in this study

\begin{tabular}{|c|c|}
\hline Primer and purpose & Sequence \\
\hline \multicolumn{2}{|c|}{ To construct pDR101 for polR disruption } \\
\hline DR Forward & GAACGCTCCTGGTCGCTCATC \\
\hline DR Reverse & ACGCCACCCTCCAGACCTACA \\
\hline \multicolumn{2}{|c|}{ To construct pSER for complementation } \\
\hline polR Forward & TCAGTGGCCGGAACGCCT \\
\hline polR Reverse & GCCGGTGGACGATGTTCG \\
\hline \multicolumn{2}{|l|}{ For RT-PCR analysis } \\
\hline RTS-polR & ACGTGAGATCCAGGAAGG \\
\hline RTA-polR & GCAACGGAAGTACACCAG \\
\hline RTS-polY & CCTCTACCGCAGTCTCCT \\
\hline RTA-polY & TGTCCTCGTCCTCCAGTT \\
\hline RTS-polC & AAGCACTACCGGCTCTGG \\
\hline RTA-polC & CTCCTTCTTTCGGGCATC \\
\hline RTS-polD & CGTGGTGAGCGACCTGTAC \\
\hline RTA-polD & AGAGGATGGCGGTGATGG \\
\hline RTS-polE & CTGCGAGGACGACGAGAA \\
\hline RTA-polE & GGCGAAGAAGGCGATGTG \\
\hline RTS-polf & CGCCTTCCAGACCGTCTACC \\
\hline RTA-polF & GGTGTCCTCGTCGCTGACCT \\
\hline RTS-polG & GCAGGAGCTGATCGTGGTC \\
\hline RTA-polG & TCGGAGTAGGGCTGGTAGG \\
\hline RTS-polH & ATCGGCTGCTCGTACTGC \\
\hline RTA-polH & CCTGCTGCGACTTGTGCT \\
\hline RTS-polI & TACGAGGGCTGGCAGAAA \\
\hline RTA-polI & GGCCGAAGCACTTGGAGA \\
\hline RTS-polJ & ACACCCCGCCGAGCGAACT \\
\hline RTA-polJ & TGCCGACCGTGCAGCAGAC \\
\hline RTS-polK & CCGGGGCAAGCACTACCT \\
\hline RTA-polK & GAGCGAACCAGCACCACC \\
\hline RTS-polL & TGGCGCAAGTACGAGGAG \\
\hline RTA-polL & CGAGGCAGTCGGTGAAGA \\
\hline RTS-polM & CACCAACGTGAGCGGACT \\
\hline RTA-polM & CGACAGGCACCACAGGAC \\
\hline RTS-polN & GCGTGAGGACGTCGTCGTA \\
\hline RTA-polN & CATGTGCTGGGGTTCGGTG \\
\hline RTS-polO & CCCGTGGAACCTGTGCTT \\
\hline RTA-polO & TAGGCGGTGAGGATGTCG \\
\hline RTS-polP & GAGGTCGTCCGCATGGTC \\
\hline RTA-polP & AGCAGCAGCAGGGTGTCC \\
\hline RTS-polQ1 & GAACAGGTCGTCGGCAAGG \\
\hline RTA-polQ1 & TGTCAGGCACAGCAGGAGG \\
\hline RTS-polQ2 & GGGAAGACCTCGCTCGCC \\
\hline RTA-polQ2 & AGACGGACCACGGGAACG \\
\hline RTS-polA & CGCAGATCCAGGTGACGA \\
\hline RTA-polA & GCTGACGGGCTGTGAGAT \\
\hline RTS-polB & GCCGTCTCCATCTGTTCG \\
\hline RTA-polB & ATCTCGCCGTCGTCAAGG \\
\hline RTS-orf1 & AGGTCGTGACGCCGTAGGT \\
\hline RTA-orf1 & TGGCTCACCACGCAGAACA \\
\hline RTS-orf2 & AAGCGGAGGGTGAAGTCG \\
\hline RTA-orf2 & CATCCCCAAGGAGATGGC \\
\hline RTS-orf3 & ATGACGAGCAGCACCACG \\
\hline RTA-orf3 & CTCCCCGACATCTCACCC \\
\hline RTS-orf4 & GTCGTCCTCCTGCGTGAA \\
\hline RTA-orf4 & GGCGTGGCTCGACTACTT \\
\hline RTS-orf5 & CCGCTCGCTCCACATCAAC \\
\hline RTA-orf5 & AGCCAGGAGTGGGTGAGGT \\
\hline
\end{tabular}


Table 1. cont.

\begin{tabular}{|ll|}
\hline Primer and purpose & \multicolumn{1}{c|}{ Sequence } \\
\hline RTS-orf6 & GGTGTTGTCGTCGTCGCA \\
RTA-orf6 & CTTCGGTCCTCGTGGCTC \\
hrdB Forward & GCCGACCCGGTCAAGGACTAC \\
hrdB Reverse & CACCGGGATACGGATGGTGC \\
For S1 nuclease mapping & \\
S-polR-SE & CGTCGAAGATGAGACATGTAGGTC \\
S-polR-AN & ATGTGTCGATCCATGTCTTCCCAC \\
S-polC-SE & GCCGGTAGTGCTTGTCGA \\
S-polC-AN & TCGACGACATGGGACACC \\
S-polB-SE & TCGTCGTTGGCGTCTTCACC \\
S-polB-AN & ATCTCCCACGCCCGTGACCT \\
For PolR overexpression in $\boldsymbol{E}$. coli & \\
PolRN & TCTCATATGATGTATGCAACGGAAGT \\
PolRC & ATAGAATTCGTGGCCGGAACGCCTGG \\
\hline
\end{tabular}

mid was further confirmed by DNA sequencing and then introduced into E. coli BL21(DE3) for protein overexpression. PolR protein was expressed as an $\mathrm{N}$-terminally $\mathrm{His}_{6}$-tagged fusion protein. E. coli BL21(DE3) carrying pET28a ::polR was grown in $200 \mathrm{ml} \mathrm{LB}$ to an $\mathrm{OD}_{600}$ of 0.6 at $37{ }^{\circ} \mathrm{C}$ and was induced with $0.1 \mathrm{mM}$ IPTG at $20{ }^{\circ} \mathrm{C}$ overnight. The cells were harvested by centrifugation and resuspended in binding buffer $\left(50 \mathrm{mM} \mathrm{NaH} \mathrm{PO}_{4}, 300 \mathrm{mM} \mathrm{NaCl}, 10 \mathrm{mM}\right.$ imidazole $\mathrm{pH}$ 8.0) and disrupted by sonication. The cleared lysate was applied to a nickel-NTA column (Qiagen). The His ${ }_{6}$-tagged PolR protein eluted with $250 \mathrm{mM}$ imidazole was analysed by SDS-PAGE. Further desalting and concentration were performed with a Microcon YM-30 centrifugal filter unit (Millipore).

Electrophoretic mobility-shift assays. The following DNA fragments were used in gel mobility-shift assays: (1) the 429 bp polC promoter fragment amplified using genomic DNA as template and the primer pair S-polC-SE/S-polC-AN; (2) the 384 bp polB promoter fragment amplified using genomic DNA as template and the primer pair S-polB-SE/S-polB-AN. The unrelated DNA fragment used as a negative control was generated by PCR using genomic DNA as template and the primer pair RTS-polF/RTA-polF (corresponding to the internal coding sequence of polF). These PCR products were labelled at the $5^{\prime}$ ends with $\left[\gamma^{32} \mathrm{P}\right] \mathrm{ATP}$ and T4 polynucleotide kinase.

For electrophoretic mobility-shift assays, the binding reaction $(20 \mu \mathrm{l})$ contained $1 \mu \mathrm{g}$ poly $(\mathrm{dI}-\mathrm{dC})$, purified protein at various concentrations and a constant concentration of labelled DNA fragment

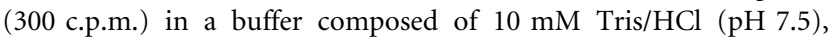
$2 \mathrm{mM}$ DTT, $5 \mathrm{mM} \mathrm{MgCl}_{2}, 0.5 \mathrm{mg}$ calf BSA ml $\mathrm{ml}^{-1}$ and $5 \%(\mathrm{v} / \mathrm{v})$ glycerol. After incubation at ambient temperature for $30 \mathrm{~min}$, DNAprotein complex and free DNA were separated by electrophoresis on non-denaturing $5 \%$ polyacrylamide gels (mono/bis, $80: 1$ ) at $6 \mathrm{~V}$ $\mathrm{cm}^{-1}$ with a running buffer of $0.5 \times$ TBE containing $40 \mathrm{mM}$ Tris/ $\mathrm{HCl}$ (pH 7.8), $20 \mathrm{mM}$ boric acid and $1 \mathrm{mM}$ EDTA. Gels were dried and exposed to Biomax radiographic film (Kodak).

\section{RESULTS}

\section{polR is a putative regulatory gene for POL biosynthesis}

The polR gene in the pol gene cluster encodes a putative polypeptide 1111 aa in length with a predicted molecular mass of $119.3 \mathrm{kDa}$. Sequence similarity search showed that PolR is most similar to several regulatory proteins including the deduced product of orfR (accession no. AJ250878) (55\% identity), a putative regulatory gene for NIK biosynthesis in S. tendae, and SanG (AY631852) (54\% identity), a pathway-specific transcriptional regulator for NIK biosynthesis in S. ansochromogenes (Liu et al., 2005). PolR also showed full-length similarity to PimR (35\% identity), a positive regulator for pimaricin biosynthesis in Streptomyces natalensis (Anton et al., 2004). All of these proteins are relatively large (1061-1198 aa) and all contain SARP-like domains in their N-termini and other similar domains in their $\mathrm{C}$-termini.

Domain prediction of PolR with SMART, Pfam and CDD confirmed that PolR has similar domain architecture to SanG and PimR (Fig. 2a). The N-terminus of PolR contains a trans-Reg-C (transcriptional regulatory Cterminal) domain (amino acids 30-110) (Pfam no. PF00486) and an accompanying BTA (bacterial transcriptional activator) domain (amino acids 117-262) (Pfam no. PF03794). This region of PolR showed significant similarity to several proteins belonging to the typical SARP family. The highest scores are AurD from the aureothin pathway in Streptomyces thioluteus (38.6\% identity) (He \& Hertweck, 2003), CcaR from the cephamycin and clavulanic acid pathways in Streptomyces clavuligerus (32.6\% identity) (Perez-Llarena et al., 1997) and RedD from the undecylprodigiosin pathway in S. coelicolor $(31.1 \%$ identity) (Narva \& Feitelson, 1990) (Fig. 2b). The C-terminus of this SARP-like region of PolR displays an AAA domain (amino acids 361-546) (SMART no. SM00382). This domain is characteristic of AAA proteins, which constitute a large family of ATPases associated with diverse cellular activities. They are thought to exert their activity through the energy-dependent unfolding of protein (Patel \& Latterich, 1998). At the sequence level, the AAA domain contains conserved Walker A and B motifs. The Walker A motif typically adopts the sequence pattern Gx4GK[T/S], 
(a)

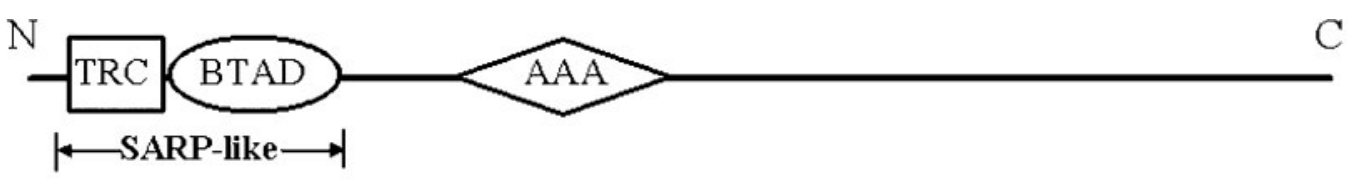

(b)

\begin{tabular}{|c|c|}
\hline POLR & ...... MYATEVHQPRTLTADLSLEAFG \\
\hline ORFR & ...... MAYTAQEQQSASL ADLSFEAFG \\
\hline SanG & . . . . MAYTAQEQQTASL TDLSFEAFG \\
\hline PimR & $\ldots \ldots \ldots \ldots$ MPAPP TANVS PATTGQAELLISVLG \\
\hline Aurd & $\ldots \ldots \ldots \ldots \ldots \ldots$ MVNAVVKLPSDLSFNILG \\
\hline $\mathrm{caR}$ & $\cdots \cdots \cdots \cdots \cdots$ \\
\hline RedD & TGR TV GRAB.DP SD HGP SL YNFGGCVE INILG \\
\hline PolR & WTSGG KD AGVAPLHYRAP \\
\hline ORFR & DSLPRRPQATLQTYLSHLRRVLTGRHG . . . P IARCATRPP \\
\hline $\operatorname{SanG}$ & D SLPRRP QP PCRTYLS HLRRVUTGRHG . . P IA PL RYQAP \\
\hline PimR & ALQSHVS HLRRALH. TVTEMGQLSVL RHR TP \\
\hline urd & GPPGNAVA SLHSYVS HLRRCLEPD CP PWGRNR IL RRE PQ \\
\hline CeaR & PP PL TVSGQLQTS AWMIRT AL AEAGL P. . RD AL GSHDR \\
\hline $\operatorname{RedD}$ & PED NVLNALQAHAARARKVUNERAC PERAGG IL RSVLG \\
\hline$\circ 1 \mathrm{R}$ & RAD PFLDLTS YE PLAEESARLQHLRTARVTIRAERL \\
\hline ORFR & RAD PFLDLA.ATPLA.E EA.ARLGLLRTTAVA.A QA TLLA \\
\hline SanG & RAD PFLDLAAYTPLAE EA.ARLSLLRTTAVAA QA DTLLT \\
\hline PimR & E F GHP PLSDERSRLEQIRLTAVESCXEARLA \\
\hline AurD & WRGSPYAEL GD YEPAVCEA.ARLEELRLA TL EALWEAELA \\
\hline caR & NKGP AF ADVTSSR. L RL RGETLEE ERTA AV EL RAL IDV \\
\hline $\operatorname{RedD}$ & $R G P$ AL IDAGE GRRC RGAA ALFEE RRLT AL EDL I SBMI \\
\hline PolR & SEELGVAPGDDLRRVHA \\
\hline ORFR & SEELGVA PA RELQRVHL ALLRHEL DDQTS. \\
\hline Sang & SEELGVA PARELQRVHLALIRHEL DDQTS . \\
\hline PimR & E RTRS HLVEE FGVD TAAELRRVRTA IU RQEP GASAPSK \\
\hline AurD & VYNR I RKATHOEL GVD P GRLRSLFAR IURD ES $\ldots \ldots$ \\
\hline CeaR & VFHRA KD IUREQ IGIS PGERMTRVMQA IURQDEQ . . . \\
\hline$=d D$ & RLARKRLDDELGVQPGALL \\
\hline
\end{tabular}

(c)

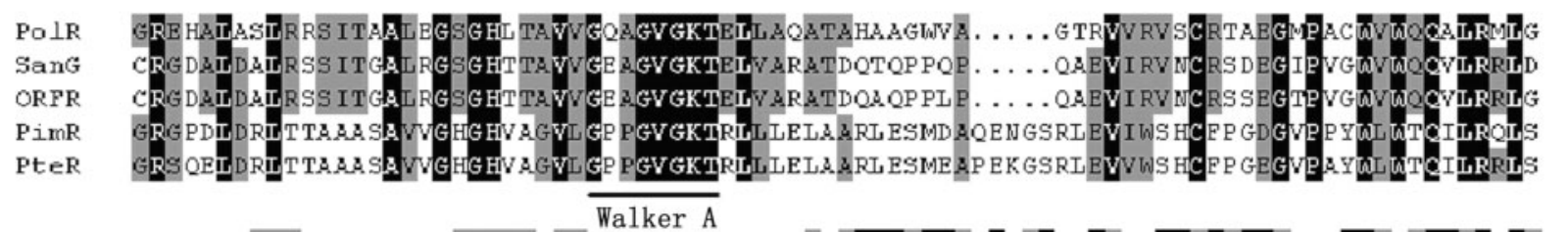

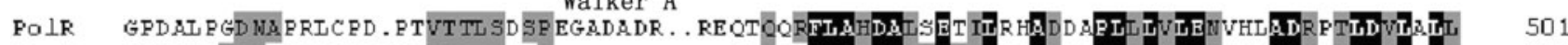

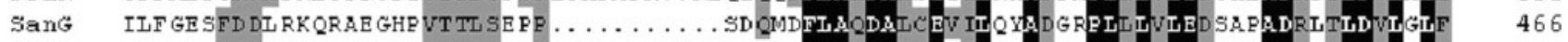

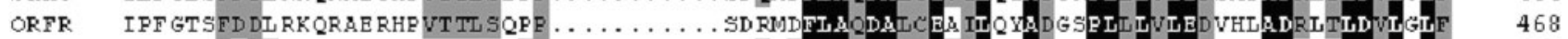

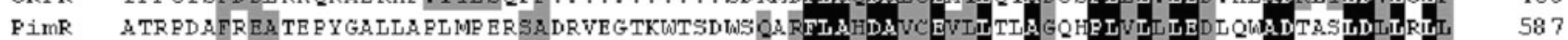

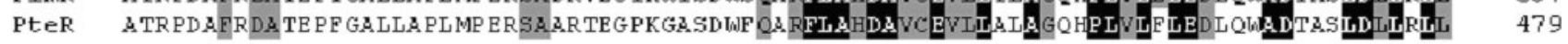

$\overline{\text { Walker B }}$

Fig. 2. Domain structure and amino acid alignment of parts of the PolR protein. (a) Predicted domain structure of PolR. TRC, trans-Reg-C domain (Pfam no. PF00486); BTAD, bacterial transcriptional activator domain (Pfam no. PF03794); AAA, domain characteristic of ATPases associated with a variety of cellular activities (SMART no. SM00382). (b) Alignment of the SARP-like domain of PolR with related proteins. Numbers indicate amino acid residues from the $\mathrm{N}$-terminus of the protein. Identical amino acid residues are highlighed in black, and similar residues in grey. ORFR, deduced product of orfR (AJ250878) from Streptomyces tendae; SanG, NIK biosynthesis regulator from S. ansochromogenes (AAV31783); PimR, pimaricin biosynthesis regulator from S. natalensis (CAE51066); AurD, regulator of the aureothin pathway in S. thioluteus (CAE02599); CcaR, activator of both the cephamycin and clavulanic acid pathways in S. clavuligerus (CAB03623); RedD, undecylprodigiosin activator in S. coelicolor (P16922). (c) Comparison of the AAA domain of PoIR with those of other proteins. The Walker A and B motifs are shown. Numbers indicate amino acid residues from the $\mathrm{N}$-terminus of the protein. Identical amino acid residues are highlighed in black, and similar residues in grey. PteR, a putative regulatory protein from S. avermitilis (NP_821585). 


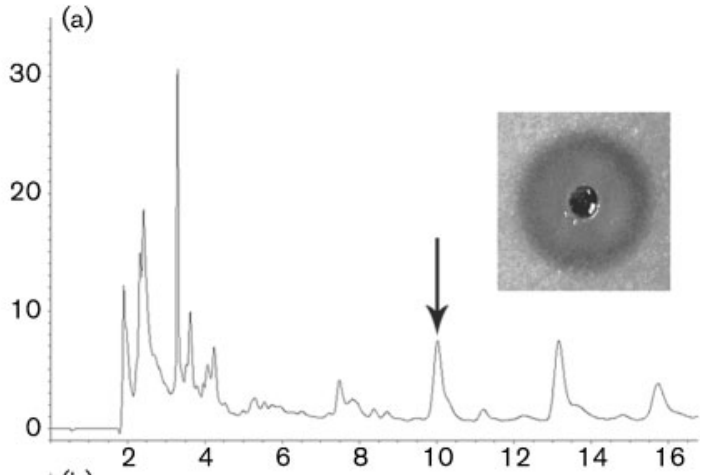

(b)

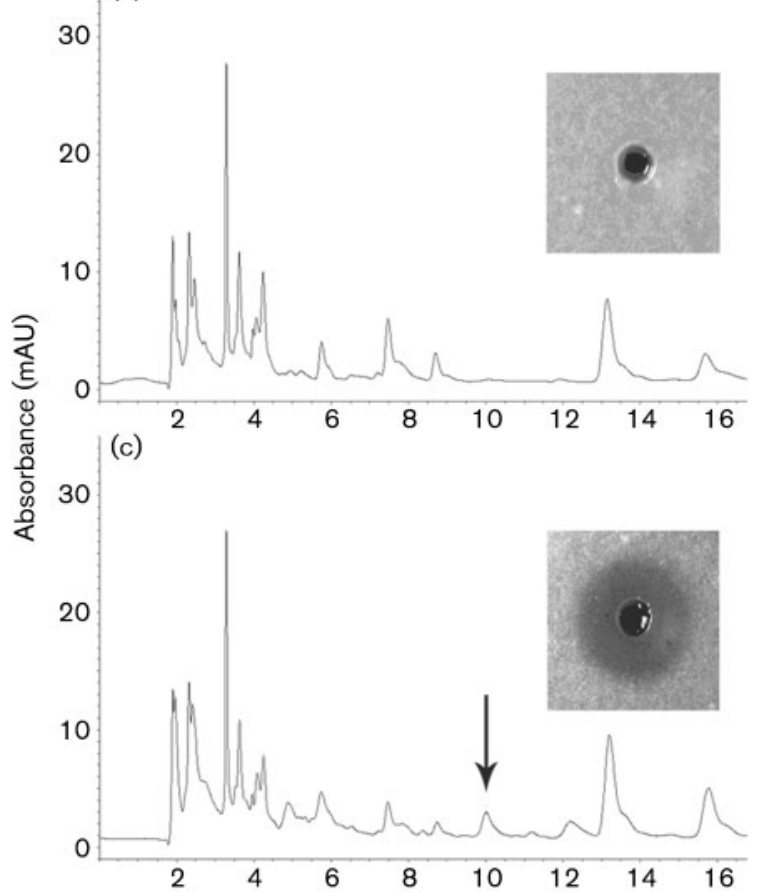

(d)

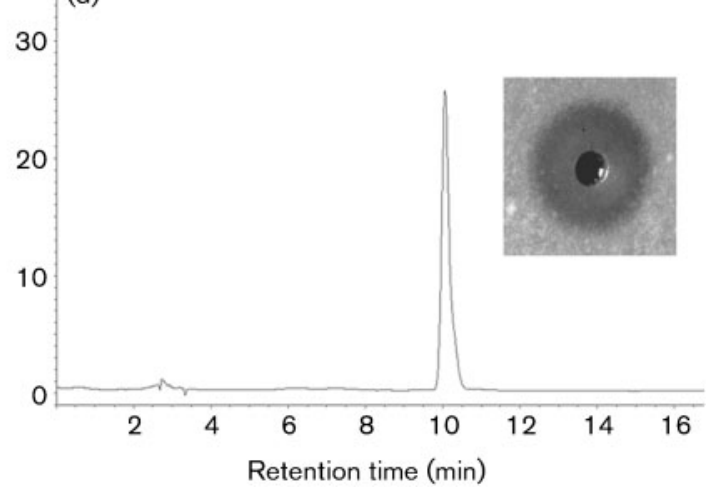

Fig. 3. HPLC analysis and bioassay of POL production. (a) Culture filtrates from the wild-type strain; (b) culture filtrates from the polR disruption mutant (DMR); (c) culture filtrates from the complemented strain (DMR/pSER); (d) POL-D standard sample. The arrows indicate the peak corresponding to POL-D. mAU, milliabsorbance units. where $\mathrm{x}$ is any residue. The Walker $\mathrm{B}$ motif is composed of a conserved aspartate (or, less commonly, glutamate) immediately after a hydrophobic strand (Leipe et al., 2003) (Fig. 2c). The C-terminal part of PolR has several dispersed low-complexity regions without known motifs.

Like many pathway-specific regulator genes in Streptomyces, polR contains a rare TTA codon (codon 64). This indicates that the expression of polR may depend on the bldA gene, which encodes a tRNA responsible for translating TTA (UUA in mRNA) into leucine (Leskiw et al., 1991).

\section{The polR mutant has lost the ability to produce POLs and polR complementation restores POL production}

To determine the role of polR, a polR disruption mutant was constructed via homologous recombination. The disruption of polR was confirmed by Southern blot analysis using a truncated fragment of $p o l R$ as a probe (data not shown). The resulting polR disruption mutant was designated DMR.

DMR and wild-type strains of $S$. cacaoi subsp. asoensis were tested for POL production using A. longipes as an indicator strain in bioassay. A large zone of growth inhibition was observed with the culture filtrates of the wild-type strain (Fig. 3a), whereas no growth-inhibiting activity against $A$. longipes was detected for DMR (Fig. 3b). This result indicated that the DMR strain had lost the ability to produce active POLs. The inability of DMR to synthesize POLs was further confirmed by HPLC. No peak corresponding to POL-D standard sample was present in the culture filtrates of DMR, but a distinct peak corresponding to POL-D was clearly exhibited in the culture filtrates of the wild-type strain (Fig. 3). The DMR strain showed growth and morphology identical to those of the wild-type on MS or R2YE agar media, indicating that polR has no significant effect on growth and differentiation of S. cacaoi subsp. asoensis.

POL production by the DMR strain was restored by introducing pSER, although at a reduced level compared to the wild-type strain (Fig. 3c). This result further confirmed that the abolition of POL production in DMR was due to polR disruption, suggesting that PolR plays a key role as a positive regulator of POL biosynthesis in S. cacaoi subsp. asoensis. The incomplete restoration of POL production by introducing pSER into strain DMR could be due to the $\phi \mathrm{C} 31$ attB site on the chromosome not being neutral for POL production. In fact, when the empty vector, pSET152, was introduced into the wild-type strain, the resulting transformants showed up to $30 \%$ reduction in POL production (data not shown).

\section{An additional copy of polR leads to overproduction of POL}

It has been reported that overexpression of pathwayspecific activators can lead to overproduction of the 
corresponding antibiotics (Chater, 1990). In our study, pSER constructed for gene complementation was used to increase gene dosage in the wild-type strain. When pSER was integrated into the $\phi \mathrm{C} 31 \mathrm{attB}$ site in the chromosome of the wild-type strain, POL production was increased about threefold in comparison with that of the wild-type strain carrying pSET152 (Fig. 4a), although the biomass of these strains was similar (Fig. 4b). This result indicated that POL biosynthesis in S. cacaoi subsp. asoensis is limited by the availability of PolR, and also reinforced the evidence that PolR is a positive regulator for POL production.

\section{The transcription start point of polR}

To determine the tsp of polR, an S1 nuclease protection assay was performed. Total RNAs isolated from S. cacaoi subsp. asoensis were hybridized with ${ }^{32} \mathrm{P}$-labelled probe (see Methods; Table 1). The result showed that polR has a single tsp, which is localized at the nucleotide $\mathrm{C}$ at position $38 \mathrm{bp}$ upstream from the polR translation start codon (Fig. 5a, b).
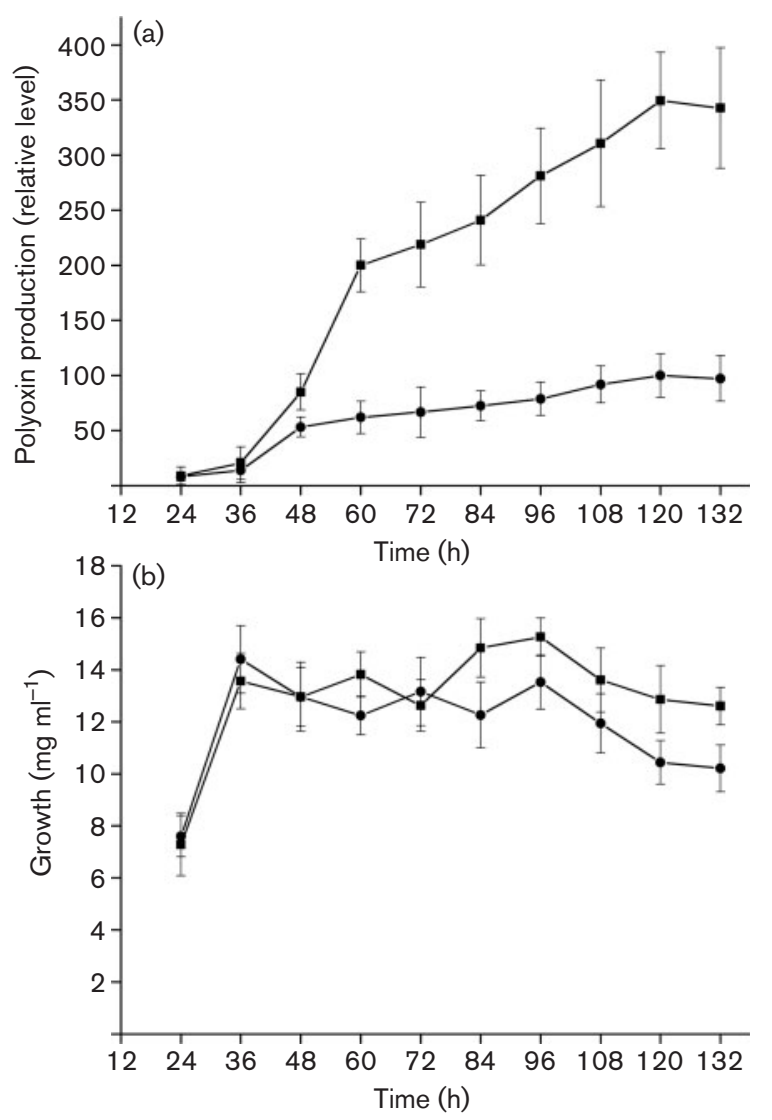

Fig. 4. Growth and POL production of $S$. cacaoi subsp. asoensis containing pSER. POL yields were normalized relative to that of the wild-type strain containing pSET152 alone (120 h fermentation), which was set at $100 \%$. (a) POL production; (b) growth curves. Wild-type strain with pSET152; $\mathbf{a}$, transformants with pSER (pSET152: : polR). Data are the means \pm SD of three duplicate flasks.
Furthermore, a region in the promoter of polR shows similarity to the consensus ARE sequence (Folcher et al., 2001), a potential target site for a $\gamma$-butyrolactone receptor (Fig. 5c), suggesting that transcription of polR might be regulated by a $\gamma$-butyrolactone receptor involved in the hierarchy of $\gamma$-butyrolactone regulation (Horinouchi, 2007).

\section{Gene expression analysis in the wild-type and strain DMR}

Because of the similarity of the N-termini of PolR with SARP-family regulators, it was possible that the transcription of some pol genes might be regulated by PolR. In order to determine whether these genes are potential targets of PolR, total RNAs were isolated from the $S$. cacaoi subsp. asoensis wild-type and DMR strains after fermentation for $48 \mathrm{~h}$ (when POL is actively produced) and used as templates for analysis of gene expression by RT-PCR. Primers for RT-PCR were designed to detect specific sequences of pol genes (Table 1). A primer pair designed to amplify cDNA of $h r d B$, encoding an essential sigma factor (accession no. EU123870) in S. cacaoi subsp. asoensis, was used as an internal control.

Among the genes of the pol cluster, seven genes (polA, polB, polD, $p o l H-K)$ were expected to encode the enzymes responsible for the biosynthesis and modification of the nucleoside moiety; eight genes (polL-P and polC, polE and polF) might encode enzymes responsible for the biosynthesis of peptidyl moieties; pol $G$ was expected to encode the carboxylase responsible for the condensation between nucleoside and peptidyl moieties to form active POLs; and two genes (polQ1 and polQ2) are likely to be responsible for transport. A recent study has demonstrated that both PolO and PolA are required for polyoxin production. $\mathrm{PolO}$ was characterized as $\mathrm{O}$-carbamoyltransferase, which catalysed the conversion of AHV ( $\alpha$-amino- $\delta$ hydroxylvaleric acid) to ACV ( $\alpha$-amino- $\delta$-carbamoylhydroxyvaleric acid), and PolA was characterized as UMPenolpyruvyl transferase, which converted UMP and PEP (phosphoenolpyruvate) into $3^{\prime}$-EUMP (3'-enolpyruvylUMP) (Chen et al., 2009). Since PolR regulates POL production, these genes are potential targets of PolR and were selected for transcription analysis by RT-PCR.

Transcripts of the 18 structural genes (polC to polQ2, polA and $p o l B$ ) were distinctly visualized in the wild-type strain of S. cacaoi subsp. asoensis after 27 cycles of PCR, whereas no transcripts could be detected in the DMR strain under the same conditions, indicating that the transcription of these genes is probably regulated by PolR and that the promoters of these genes are likely to be the targets (directly or indirectly) of PolR.

For transcriptional analysis of polR, a pair of primers located before the insert site of the kanamycin resistance gene was used. A similar transcription pattern was observed for polR in the wild-type and DMR strains, indicating that polR was 
(a)

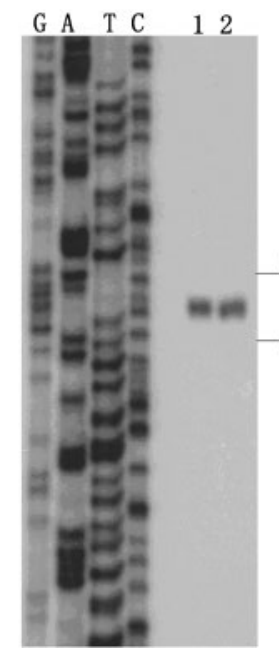

(b)

CGGCCGACGAAACTGAAATGCGCTGATCAGAGTGTATGTGGCAG

$\mathrm{ARE}$

GCTCTCCGGCAGGCCGTGGCCGCCAAGTGGAGGGAGACGTTCCT

$\overline{\text { TGGCTTGTTC }}$ TCACCGTCAGTGCAACGTCACTCTTTCCGCACAGG

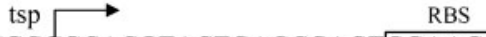

GGCATGTGTAGCCTGCTCGCCGCACGTACTGAGCGAGTGGAAGG

CATATGCACCAGGCATGATGTATGCAACGGAAGTACACCAGCCC $\begin{array}{lllllllllll}p o l R> & M & M & Y & A & T & E & V & H & Q & P\end{array}$

(c)

ARE consensus POIR

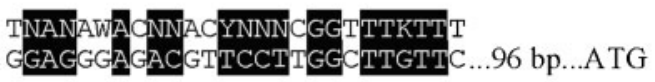

Fig. 5. Transcriptional analysis of polR. (a) High-resolution $\mathrm{S} 1$ nuclease mapping of polR. Lanes $\mathrm{G}, \mathrm{A}, \mathrm{T}$ and $\mathrm{C}$, DNA sequencing ladder; lanes 1 and 2, RNAs isolated from $S$. cacaoi subsp. asoensis after fermentation for 48 h. The asterisk indicates the tsp of polR. (b) The nucleotide sequence covering the promoter region. The ARE (autoregulatory element), a conserved DNA motif recognized by many $\gamma$-butyrolactone receptors (Folcher et al., 2001), is overlined; the tsp is indicated by an arrow; the ribosome-binding site (RBS) is boxed. (c) Alignment of the putative $\gamma$-butyrolactone receptor binding site with the ARE consensus (Folcher et al., 2001). The distance between the ARE site and the translational start codon of polR is indicated. The letters $\mathrm{Y}, \mathrm{K}$ and $\mathrm{W}$ represent $\mathrm{C}$ or $\mathrm{T}, \mathrm{G}$ or $\mathrm{T}$, and $\mathrm{A}$ or $\mathrm{T}$, respectively.

not auto-regulated. An interesting and unexpected finding in transcript analysis was that polY transcripts could not be detected in either the wild-type or the DMR strain even when the number of PCR cycles was increased to 32 . Sequence analysis suggested that polY might be another regulatory gene in the pol cluster. Further studies need to be done to determine the hierarchy of polR and polY.

The transcription patterns of six other adjacent genes (orf-1 to orf-6 in Fig. 1b) were also analysed. They have been experimentally confirmed to be unrelated to POL biosynthesis (Chen et al., 2009). Their transcripts could not be detected in either the wild-type or DMR strains after 27 cycles of RT-PCR. When the number of PCR cycles was increased to 32 , transcripts of the six genes were detected in both strains (Fig. 6), suggesting that these six genes are expressed constitutively at a low level in vivo and their expression is independent of PolR.

\section{The presence of promoters upstream of po/C and polB}

The data presented above indicated that the expression of 18 pol genes is positively regulated by PolR. These 18 genes are probably transcribed from at least two transcriptional units based on their genetic organization (Fig. 1b), although this will require further confirmation. The 16 genes from polC to polQ2 are transcribed in the same direction. Half of these 16 genes ( $p o l G-K$ and $p o l M-O$ ) are translationally coupled as judged by their overlapping codons, and no predicted transcriptional terminator could be found in the short intergenic regions between other adjacent genes, indicating that these 16 genes might form a transcriptional unit. polB and polA are divergently transcribed and they might constitute another operon as deduced by the lack of an obvious terminator in their intergenic region.

If this is the case, polC and polB must have their own promoters since they are the first genes in the two respective putative operons. Therefore, S1 mapping experiments were carried out to determine the tsps of polC and polB. As expected, polC has a single tsp, which is localized at the nucleotide $\mathrm{C}$ (Fig. 7a). polB is also transcribed from a single promoter, and its tsp is localized at the nucleotide $\mathrm{T}$ (Fig. 7a). Interestingly, similar heptameric direct repeats (5'-CGGCAAG- $\left.3^{\prime}\right)$ with little variation were identified in the respective -35 regions of the promoters (Fig. $7 \mathrm{~b}$ ). We propose that these heptameric repeats might be the target sequences of PolR.

\section{Interaction of PoIR with the promoter regions of polB and polc}

In order to determine whether PolR directly acts on the DNA regions mentioned above, PolR was expressed as an $\mathrm{N}$-terminally $\mathrm{His}_{6}$-tagged protein in E. coli. Upon cultivation at $37{ }^{\circ} \mathrm{C}$ and induction with $0.1 \mathrm{mM}$ IPTG at $18{ }^{\circ} \mathrm{C}$ overnight, the additional protein was detected in the crude lysate of the E. coli stain carrying pET28a::polR. The 


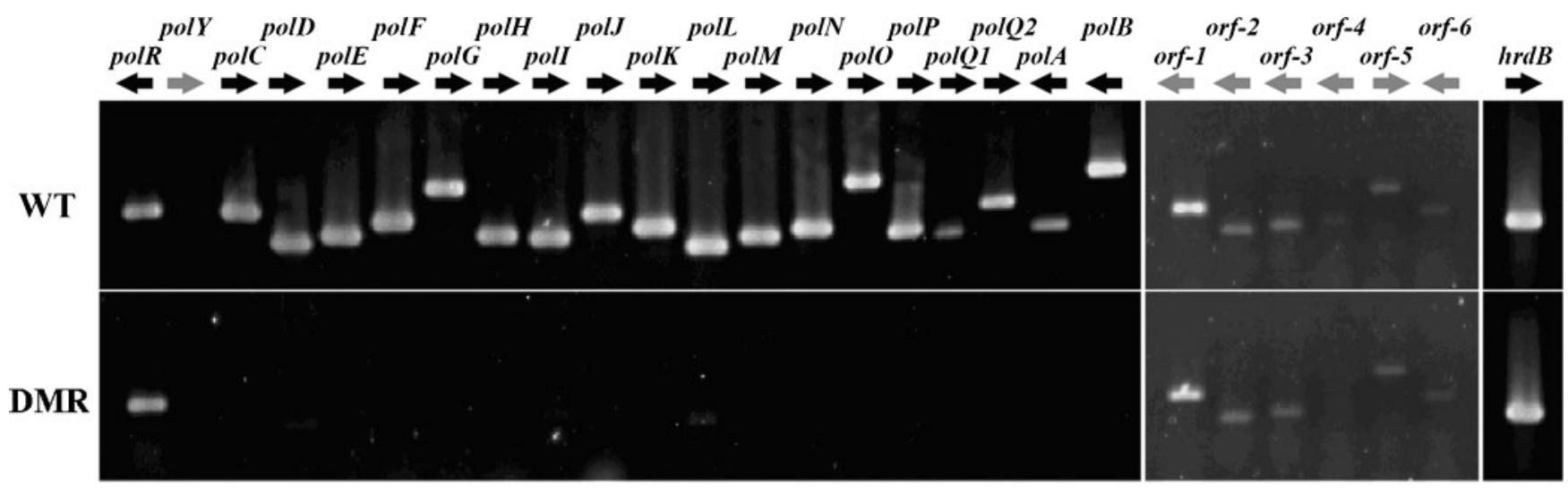

Fig. 6. Transcriptional analysis of the pol cluster by semiquantitative RT-PCR. The top panel shows the RT-PCR products from the RNAs of the wild-type strain (WT); the bottom panel shows the RT-PCR products from the RNAs of the polR disruption mutant (DMR). Total RNAs were isolated from mycelia after fermentation for $48 \mathrm{~h}$; hrdB transcript was used as an internal control. Twenty-seven cycles of PCR were routinely employed (genes represented by black arrows); whenever no product was detected, 32 cycles of PCR (genes represented by grey arrows) were repeated to detect low-level transcripts.

supernatant fraction was applied to an Ni-affinity resin column, from which $\mathrm{N}$-terminally $\mathrm{His}_{6}$-tagged PolR was purified. SDS-PAGE analysis showed a band of about $120 \mathrm{kDa}$, which is similar to the expected size of PolR (Fig. 8a).

Gel mobility-shift assays were carried out using the purified PolR and PCR-amplified DNA promoter regions of polC and $p o l B$, respectively. The $429 \mathrm{bp}$ fragment containing the polC promoter region was located at position $-257 \mathrm{bp}$ to $+172 \mathrm{bp}$ with respect to the tsp of polC. The $384 \mathrm{bp}$ fragment containing the polB promoter region was located at position $-205 \mathrm{bp}$ to $+179 \mathrm{bp}$ with respect to the tsp of polB. As shown in Fig. 8(b), purified PolR showed clear binding to both labelled promoter fragments ( $p o l C$ and polB probes). To examine the binding specificity, a $424 \mathrm{bp}$ DNA fragment corresponding to an internal coding sequence of polF was used as a negative control. Purified PolR did not display binding activity with the unrelated DNA fragment (polF probe) (Fig. 8b). These results demonstrated that PolR protein has a specific binding activity for the promoter regions of polC and polB. (a)
polC

G A T C 12

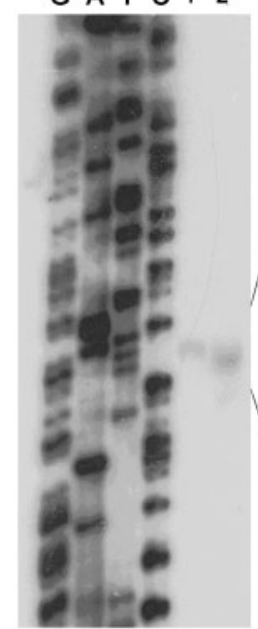

polB

GA TC 12

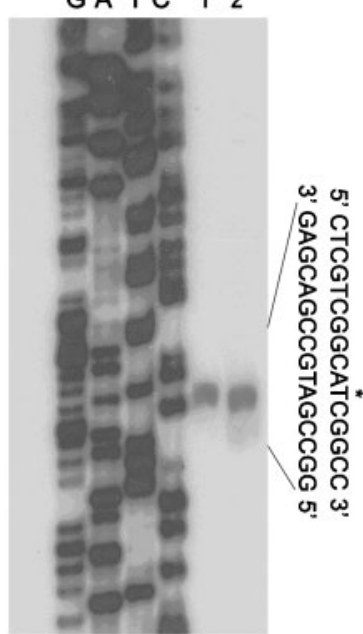

(b)

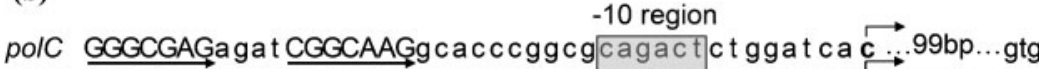
polB GGGCAAGgaccCGGCAGGaaggcggccccacact cgt cggca t ...58bp...atg
Fig. 7. Determination of the tsps of po/C and polB. (a) Transcriptional analysis of $p o / C$ and polB by $\mathrm{S} 1$ mapping. Lanes $\mathrm{G}, \mathrm{A}, \mathrm{T}$ and $\mathrm{C}$, DNA sequencing ladder; lanes 1 and $2, S 1$ nuclease-treated samples; the $\mathrm{S} 1$ nuclease reactions were carried out with total RNAs isolated from $48 \mathrm{~h}$ culture of $S$. cacaoi subsp. asoensis. The tsps of $p o / C$ and $p o / B$ are indicated by asterisks. (b) Alignment of the promoter regions of $p o / C$ and $p o / B$. The $7 \mathrm{bp}$ direct repeats and -10 regions in the cognate promoter sequences are compared. The tsps and transcription direction of $p o / C$ and $p o / B$ are indicated with bold letters and bent arrows. The distance between a tsp and the start codon of the downstream gene is indicated. 


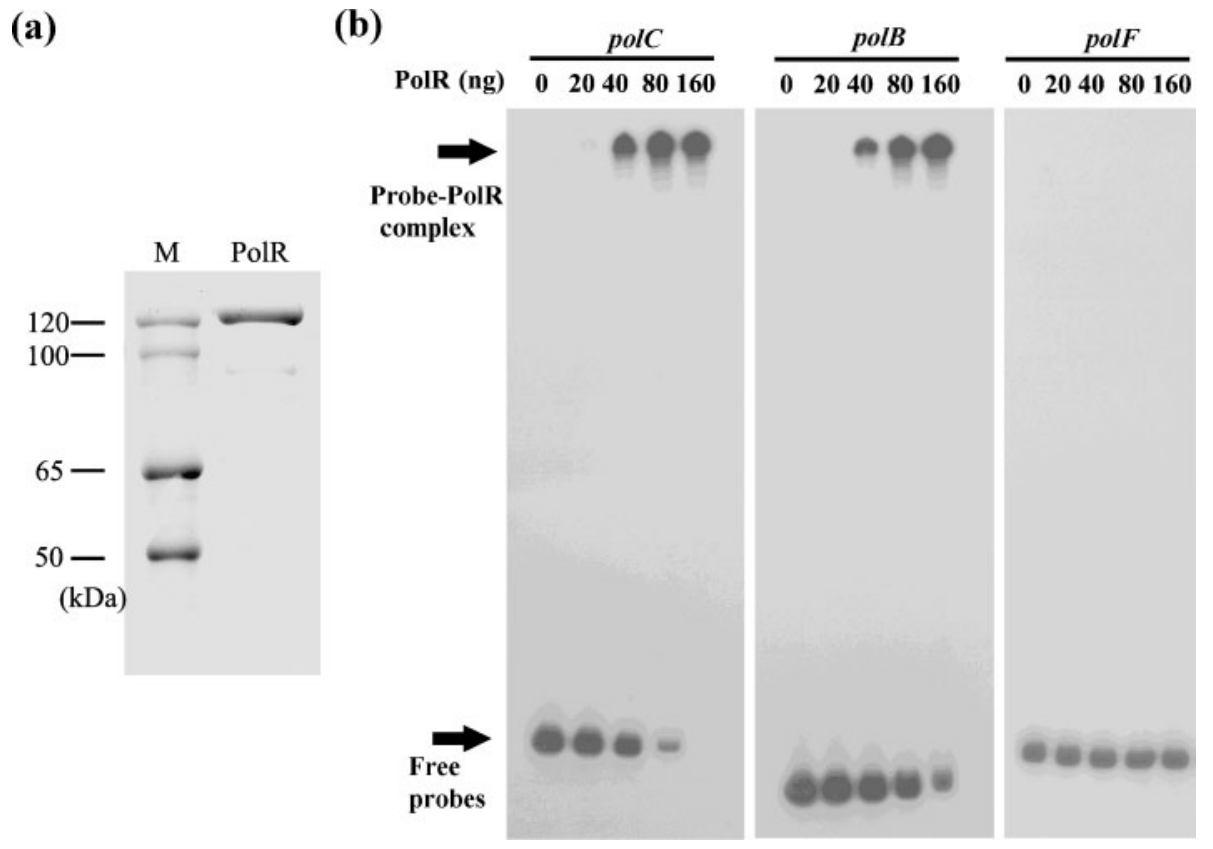

Fig. 8. Binding of purified PolR to the promoter regions of po/C and polB. (a) SDS-PAGE analysis of purified PolR. M, molecular mass markers. (b) PolR binds to the promoter regions of po/C and polB. An increasing amount of purified PolR was added to the labelled $p o / C$ and polB probes; an internal fragment of polF coding sequence was used as a negative control. DNA-protein complexes and free probes are indicated by arrows.

\section{DISCUSSION}

The genetic analysis of the biosynthetic pathway for the two 'classical' nucleoside peptide antibiotics, namely NIKs and POLs, revealed that 12 genes in the pol cluster are likely to be shared by the nik gene cluster. This is not surprising, since the structures of these antibiotics share some similar features. The evidence that polR in the pol cluster is essential for POL production, like its counterpart in the nik cluster, $\operatorname{san} G$, which is essential to activate NIK production, strongly suggested that similar regulatory cascades exist in both POL and NIK biosynthesis. The finding of the rare TTA codon in both polR and san $G$ implies that they are both translationally controlled by bldA, the structure gene of tRNA ${ }^{\mathrm{UUA}}$ (Leskiw et al., 1991; Liu et al., 2005). Furthermore, the conserved binding sites of the $\gamma$ butyrolactone receptor are present in the promoter regions of both polR and $\operatorname{san} G$, suggesting that they are likely to be transcriptionally regulated by a $\gamma$-butyrolactone receptorlike regulator in vivo (Folcher et al., 2001; Liu et al., 2005). Despite the similarities mentioned above, PolR and SanG also showed different regulatory patterns. In POL biosynthesis, PolR positively regulates the genes responsible for the synthesis of both the peptidyl moiety and the nucleotide skeleton, while in NIK biosynthesis, SanG controls only the expression of the $\operatorname{san} O$ and $\operatorname{sanN}$ operons, which contain the genes mainly responsible for the biosynthesis of the peptidyl moiety (Liu et al., 2005). In addition, PolR in S. cacaoi subsp. asoensis only affects POL production, while SanG in S. ansochromogenes influences both NIK production and colony development (Liu et al., 2005).

RT-PCR analysis revealed that the disruption of polR impaired the expression of 18 structural genes in the pol cluster, implying that the potential targets of PolR might exist in these genes. Based on the organization of the pol gene cluster, these 18 genes are probably transcribed from at least two transcriptional units. Therefore, the promoter regions of polC and polB may contain the binding sites of PolR. Gel mobility-shift assays demonstrated that PolR does bind to the promoter regions of polB and polC. We have determined the tsps of polC and polB, and their deduced promoters showed a similar structure. Both of them contain heptameric direct repeats (consensus: $5^{\prime}-$ CGGCAAG-3'), which overlap with the -35 region of the corresponding promoter, and the distance between the centres of the direct repeats is $11 \mathrm{bp}$, corresponding to one complete turn of DNA helix (Fig. 7b). These promoter characteristics are well consistent with the common features of promoters recognized by SARP-family proteins (Tanaka et al., 2007). Considering the similarity of the Nterminal section of PolR with proteins of the SARP family, it is likely that PolR follows a regulatory pattern similar to those of SARPs and activates transcription of the polC and polB operons by binding to the heptameric direct repeats. Domain prediction indicates that PolR has an ATPase domain in addition to the SARP-like domain. Thus, the functions of the ATPase domain and the C-terminal part of PolR are interesting topics for further investigation. 
Domain prediction indicates that PolY consists of an Nterminal SARP-like domain, a central ATPase domain and a C-terminal tetratricopeptide repeat (TPR) domain. The domain composition of PolY is identical to that of AfsR (Horinouchi, 2003). Gene disruption experiments indicated that $p o l Y$ is also indispensable for POL production (our unpublished data). Unexpectedly, polY transcripts could not be detected in either the wild-type or the DMR strain after incubation for $48 \mathrm{~h}$. Further experimental analysis in progress will hopefully elucidate the exact role of polY in POL biosynthesis.

The similarity between the biosynthetic gene clusters of POL and NIK provides an opportunity to carry out combinatorial biosynthesis for production of novel nucleoside peptide antibiotics. Further understanding of PolR will not only be helpful for construction of engineered strains with higher production of known metabolites, but will also facilitate the detection of novel compounds resulting from combinatorial biosynthesis by improving the expression of genes involved in secondary metabolism (Hranueli et al., 2005).

\section{ACKNOWLEDGEMENTS}

This work was supported by grants from the Ministry of Science and Technology of China (Grant nos 2009CB118905 and 2006AA10A209). We thank Dr Wenbo Ma (University of California, Riverside, USA) for critical reading of the manuscript.

\section{REFERENCES}

Anton, N., Mendes, M. V., Martin, J. F. \& Aparicio, J. F. (2004). Identification of $\mathrm{PimR}$ as a positive regulator of pimaricin biosynthesis in Streptomyces natalensis. J Bacteriol 186, 2567-2575.

Arias, P., Fernandez-Moreno, M. A. \& Malpartida, F. (1999). Characterization of the pathway-specific positive transcriptional regulator for actinorhodin biosynthesis in Streptomyces coelicolor A3(2) as a DNA-binding protein. J Bacteriol 181, 6958-6968.

Bierman, M., Logan, R., O’Brien, K., Seno, E. T., Rao, R. N. \& Schoner, B. E. (1992). Plasmid cloning vectors for the conjugal transfer of DNA from Escherichia coli to Streptomyces spp. Gene 116, 43-49.

Bormann, C., Mohrle, V. \& Bruntner, C. (1996). Cloning and heterologous expression of the entire set of structural genes for nikkomycin synthesis from Streptomyces tendae Tu901 in Streptomyces lividans. J Bacteriol 178, 1216-1218.

Bruntner, C., Lauer, B., Schwarz, W., Mohrle, V. \& Bormann, C. (1999). Molecular characterization of co-transcribed genes from Streptomyces tendae Tu901 involved in the biosynthesis of the peptidyl moiety of the peptidyl nucleoside antibiotic nikkomycin. Mol Gen Genet 262, 102-114.

Chater, K. F. (1990). The improving prospects for yield increase by genetic engineering in antibiotic producing Streptomyces. Biotechnology 8, 115-121.

Chen, W., Zeng, H. \& Tan, H. (2000). Cloning, sequencing, and function of sanF: a gene involved in nikkomycin biosynthesis of Streptomyces ansochromogenes. Curr Microbiol 41, 312-316.

Chen, W., Huang, T., He, X., Meng, O., You, D., Bai, L., Li, J., Wu, M., $\mathrm{Li}$, R. \& other authors (2009). Characterization of the polyoxin biosynthetic gene cluster from Streptomyces cacaoi and engineered production of polyoxin H. J Biol Chem 284, 10627-10638.

Endo, A., Kakiki, K. \& Misato, T. (1970). Mechanism of action of the antifungal agent polyoxin D. J Bacteriol 104, 189-196.

Folcher, M., Gaillard, H., Nguyen, L. T., Nguyen, K. T., Lacroix, P., Bamas-Jacques, N., Rinkel, M. \& Thompson, C. J. (2001). Pleiotropic functions of a Streptomyces pristinaespiralis autoregulator receptor in development, antibiotic biosynthesis, and expression of a superoxide dismutase. J Biol Chem 276, 44297-44306.

He, J. \& Hertweck, C. (2003). Iteration as programmed event during polyketide assembly; molecular analysis of the aureothin biosynthesis gene cluster. Chem Biol 10, 1225-1232.

Hopwood, D. A., Chater, K. F. \& Bibb, M. J. (1995). Genetics of antibiotic production in Streptomyces coelicolor A3(2), a model streptomycete. Biotechnology 28, 65-102.

Horinouchi, S. (2003). AfsR as an integrator of signals that are sensed by multiple serine/threonine kinases in Streptomyces coelicolor A3(2). $J$ Ind Microbiol Biotechnol 30, 462-467.

Horinouchi, S. (2007). Mining and polishing of the treasure trove in the bacterial genus Streptomyces. Biosci Biotechnol Biochem 71, 283299.

Hranueli, D., Cullum, J., Basrak, B., Goldstein, P. \& Long, P. F. (2005). Plasticity of the Streptomyces genome - evolution and engineering of new antibiotics. Curr Med Chem 12, 1697-1704.

Isono, K. (1988). Nucleoside antibiotics: structure, biological activity, and biosynthesis. J Antibiot (Tokyo) 41, 1711-1739.

Isono, K., Asahi, K. \& Suzuki, S. (1969). Studies on polyoxins, antifungal antibiotics. 13. The structure of polyoxins. J Am Chem Soc 91, 7490-7505.

Kieser, T., Bibb, M. J., Buttner, M. J., Chater, K. F. \& Hopwood, D. A. (2000). Practical Streptomyces Genetics. Norwich, UK: John Innes Foundation.

Kuhstoss, S., Richardson, M. A. \& Rao, R. N. (1991). Plasmid cloning vectors that integrate site-specifically in Streptomyces spp. Gene 97, 143-146.

Lauer, B., Sussmuth, R., Kaiser, D., Jung, G. \& Bormann, C. (2000). A putative enolpyruvyl transferase gene involved in nikkomycin biosynthesis. J Antibiot (Tokyo) 53, 385-392.

Lauer, B., Russwurm, R., Schwarz, W., Kalmanczhelyi, A., Bruntner, C., Rosemeier, A. \& Bormann, C. (2001). Molecular characterization of cotranscribed genes from Streptomyces tendae Tu901 involved in the biosynthesis of the peptidyl moiety and assembly of the peptidyl nucleoside antibiotic nikkomycin. Mol Gen Genet 264, 662-673.

Leipe, D. D., Koonin, E. V. \& Aravind, L. (2003). Evolution and classification of P-loop kinases and related proteins. J Mol Biol 333, 781-815.

Leskiw, B. K., Lawlor, E. J., Fernandez-Abalos, J. M. \& Chater, K. F. (1991). TTA codons in some genes prevent their expression in a class of developmental, antibiotic-negative, Streptomyces mutants. Proc Natl Acad Sci U S A 88, 2461-2465.

Liu, G., Tian, Y., Yang, H. \& Tan, H. (2005). A pathway-specific transcriptional regulatory gene for nikkomycin biosynthesis in Streptomyces ansochromogenes that also influences colony development. Mol Microbiol 55, 1855-1866.

Müller, H., Furter, R., Zähner, H. \& Rast, D. M. (1981). Metabolic products of microorganisms. Arch Microbiol 130, 195-197.

Narva, K. E. \& Feitelson, J. S. (1990). Nucleotide sequence and transcriptional analysis of the redD locus of Streptomyces coelicolor A3(2). J Bacteriol 172, 326-333.

Paradkar, A. S., Aidoo, K. A. \& Jensen, S. E. (1998). A pathwayspecific transcriptional activator regulates late steps of clavulanic 
acid biosynthesis in Streptomyces clavuligerus. Mol Microbiol 27, 831-843.

Patel, S. \& Latterich, M. (1998). The AAA team: related ATPases with diverse functions. Trends Cell Biol 8, 65-71.

Perez-Llarena, F. J., Liras, P., Rodriguez-Garcia, A. \& Martin, J. F. (1997). A regulatory gene $(c c a R)$ required for cephamycin and clavulanic acid production in Streptomyces clavuligerus: amplification results in overproduction of both beta-lactam compounds. J Bacteriol 179, 2053-2059.

Stutzman-Engwall, K. J., Otten, S. L. \& Hutchinson, C. R. (1992).

Regulation of secondary metabolism in Streptomyces spp. and overproduction of daunorubicin in Streptomyces peucetius. J Bacteriol 174, 144-154.

Tanaka, A., Takano, Y., Ohnishi, Y. \& Horinouchi, S. (2007). AfsR recruits RNA polymerase to the afsS promoter: a model for transcriptional activation by SARPs. J Mol Biol 369, 322-333.

Wietzorrek, A. \& Bibb, M. (1997). A novel family of proteins that regulates antibiotic production in streptomycetes appears to contain an OmpR-like DNA-binding fold. Mol Microbiol 25, 1181-1184.

Edited by: L. Heide 